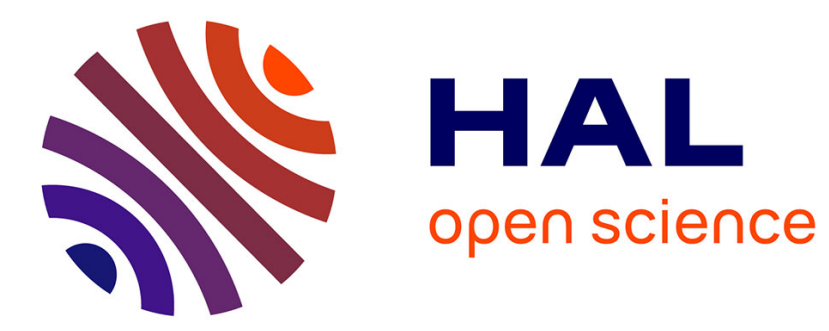

\title{
Georges Gurvitch et le droit administratif
}

Mathieu Doat

\section{To cite this version:}

Mathieu Doat. Georges Gurvitch et le droit administratif. Droit et Société, 2016, Nº 94 (3), pp.537546. 10.3917/drs.094.0537 . hal-03182106

\section{HAL Id: hal-03182106 https://hal-univ-perp.archives-ouvertes.fr/hal-03182106}

Submitted on 1 Apr 2021

HAL is a multi-disciplinary open access archive for the deposit and dissemination of scientific research documents, whether they are published or not. The documents may come from teaching and research institutions in France or abroad, or from public or private research centers.
L'archive ouverte pluridisciplinaire HAL, est destinée au dépôt et à la diffusion de documents scientifiques de niveau recherche, publiés ou non, émanant des établissements d'enseignement et de recherche français ou étrangers, des laboratoires publics ou privés. 


\author{
Georges Gurvitch et le droit administratif \\ Mathieu Doat \\ Professeur de droit public - Université de Brest \\ Membre du Lab-LEX
}

L'œuvre de Georges Gurvitch se prête-t-elle à une lecture par la doctrine administrative? Quel usage pouvons-nous faire en droit administratif d'une sociologie juridique qui a couvert de nombreux champs mais qui n'a jamais porté sur l'administration? A ces questions, une première réponse serait naturellement qu'une lecture de Gurvitch serait aujourd'hui inutile sauf pour ceux qui aiment faire des détours.

Cette réponse négative peut se justifier du point de vue de la doctrine administrative. Si ce terme de doctrine recouvre naturellement des réalités différentes et des conceptions du droit administratif variées ${ }^{1}$, la matière est marquée dès son origine par ses caractères technique et jurisprudentiel. Le savoir des administrativistes s'est construit principalement autour des "grands arrêts » du Conseil d'Etat qui sont l'emblème de la discipline et « qui réunissent pour sa seule dissection les seuls initiés à ce rituel ésotérique $\|^{2}$. Si le droit administratif au début du $20^{\circ}$ siècle a connu de spectaculaires ouvertures vers le champ de la sociologie avec des auteurs comme Hauriou et Duguit, la doctrine s'est vite repliée sur son objet fétiche, le juge administratif. Pris dans un jeu de concurrence des savoirs sur l'appareil de l'Etat en faisant face au développement des disciplines extérieures comme la sociologie, la science politique et l'économie, le droit administratif s'est vu progressivement contester sa connaissance sur le fonctionnement de l'Etat, en subissant la concurrence du développement du droit constitutionnel et du droit international. Pour des raisons tant de stratégies que d'épistémologie, la doctrine administrativiste a rapidement tracé ses frontières, en prenant en considération essentiellement « les modèles et les symboles abstraits ", en laissant dans l'ombre le fonctionnement réel de la production juridique dans l'administration.

La non lecture de Gurvitch pourrait aussi se justifier au regard du contenu de son œuvre. Tout d'abord, interroger le droit administratif à partir d'une lecture de Gurvitch n'est pas évident car, s'il est un des auteurs clefs pour penser les droits sociaux et le pluralisme juridique, il est un domaine où son apport est moins immédiatement apparent: l'Etat et le droit que génère l'appareil étatique. Si la question de l'Etat est largement présente dans l'œuvre de Gurvitch, son approche se

\footnotetext{
${ }^{1}$ Eric Millard, Ce que " doctrine » veut dire in La doctrine en droit administratif, ouvrage issu du colloque annuel de l'AFDA, éd. LexisNexis, col. Colloques et débats, 2010, pp.3-12.

${ }^{2}$ Yves Poirmeur \& Emmanuel Fayet, La doctrine administrative et le juge administratif, la crise d'un modèle de production du droit, in Le droit administratif en mutation, (dir) J.Chevallier, PUF 1993 p.106.

${ }^{3}$ G.Gurvitch, Traité de sociologie, Introduction, PUF 1958 p.21.
} 
situe souvent en amont de l'appareil administratif, aux sources de l'institution et de la normativité. Dans le contexte historique qui est le sien, il est plus un penseur du politique, de la démocratie que de la norme. II s'intéresse plus aux fondations qu'à l'édifice. Or, cette question des fondations est moins présente dans le discours des juristes contemporains, qui renvoient pour ces problèmes, aux travaux des pères fondateurs du droit administratif.

A ce décalage, suit un obstacle, lié à la difficulté même de lire ce grand auteur. "L'abondance des métaphores $»^{4}$, une écriture parfois absconse ${ }^{5}$ et catégorique rendent le premier accès rugueux pour le juriste, généralement prudent. Le travail critique de démolition des concepts juridiques les mieux établis et des affirmations parfois péremptoires accentuent cette première impression. A la lecture de Gurvitch, on mesure l'écart dont parlait Pierre Bourdieu entre les juristes et les sociologues, "l'écart entre deux modes de production et de reproduction du savoir et plus largement entre deux systèmes de valeurs, et deux styles de vie ${ }^{6}$. Et il est certain que cette distance entre Gurvitch et les juristes français pourrait aussi s'expliquer par l'empreinte russe, l'héritage social d'un intellectuel qui dû fuir son pays et construire son projet scientifique dans une autre langue et un contexte social et politique en crise profonde.

En l'occurrence, c'est cette différence de culture de Georges Gurvitch qui semble constituer pour la science du droit et plus particulièrement pour la doctrine administrativiste une possible ressource. Afin de pouvoir prendre du champ avec le savoir technique sur l'administration et plus largement avec la doxa juridique, il importe de s'ouvrir à une autre culture juridique. Alors que le champ du droit administratif est en plein bouleversement du fait notamment de la transformation profonde de l'appareil étatique, il peut être opportun d'échapper à une certaine manière de concevoir et d'agir plus ou moins formalisée, au modelage de notre pensée, ce que les grecs appelaient de manière générale la paedeia ${ }^{7}$. Si l'héritage culturel est important pour faire un "bon " administrativiste, ce même héritage peut se révéler extrêmement encombrant pour tenter de saisir des mutations juridiques.

II convient d'adopter une posture équivalente avec le legs de Georges Gurvitch afin d'appréhender ses textes moins comme des monuments de la pensée juridique et sociologique mais comme des documents de travail qui nous sont parvenus pour écrire dessus, avec le risque naturellement de faire un « enfant dans le dos » à l'auteur.

Autrement dit, il faut essayer de tenir à distance deux sortes de mémoires, celle pratiquée par les juristes pour lesquels le droit technique est un modèle abouti ;

\footnotetext{
${ }^{4}$ Cf. Jacques Le Goff, Georges Gurvitch, Le pluralisme créateur, éd. Michalon, col. Le bien commun, 2012, p.11.

${ }^{5}$ Voir notamment, l'ouvrage difficile de Gurvitch, Morale théorique et science des mœurs, PUF $2^{\circ}$ éd. 1948.

${ }^{6}$ Pierre Bourdieu, Homo academicus, éd. de Minuit, 1984, p.82.

${ }^{7}$ Le mot paedeia ou paideia ( $\pi \alpha\llcorner\delta \varepsilon i \alpha$ ) signifie " éducation » ou " élevage d'enfant ». Historiquement, il fait référence à un système d'instruction de l'ancienne Athènes. Sur ce terme et plus largement, sur l'opposition entre culture et méthode, voir Roland Barthes, Comment vivre ensemble, in Cours au collège de France du 12 janvier 1977, enregistrement CD. Ed. du Seuil 1989. Pour une approche juridique, voir Mickael Lavaine, L'acte juridictionnel en droit administratif français, thèse dactyllographié, université de Brest 2015, pp.14-18.
} 
celle sur Georges Gurvitch dont la stature pourrait empêcher d'avoir un rapport critique avec son œuvre, au moment de la commémoration de sa disparition.

Comme l'a montré Jacques Caillosse à propos de Pierre Bourdieu, « il doit bien être possible de faire du texte du sociologue un espace à l'intérieur duquel une réflexion juridique sur l'identité du droit continue de se développer librement, sans qu'il lui faille acquitter des "droits de douane " coûteux au point de rendre totalement dissuasif le passage de la frontière disciplinaire $»^{8}$.

Sur ce chemin difficile, de nombreuses approches sont possibles tant l'œuvre de Gurvitch est riche. Sans prétendre à une analyse complète, il convient de tenter brièvement de nous engager en essayant de suivre deux lignes directrices qui se recoupent. La première nous amène très naturellement sur un terrain classique que connaît tout sociologue : le rapport du droit au réel. II est incontestable que Gurvitch a jeté sur la production juridique et sur l'effectivité des droits, des lumières décisives. Et c'est en suivant cette première ligne que nous croiserons la question du conflit, une thématique plus en creux dans l'œuvre de Gurvitch mais tout aussi essentielle.

\section{I - Du retour au réel : le fait normatif administratif}

Lorsque Georges Gurvitch quitta la Russie pour venir en France en 1925, après avoir séjourné cinq ans, à Prague, un éloignement net s'opérât avec les textes juridiques. Les faiblesses et les insuffisances d'une approche juridique, pour l'étude des transformations de la société apparurent progressivement. Les rapports sociaux pouvaient d'autant moins être appréhendés principalement à travers la lecture des textes juridiques, que les soubresauts des Etats brouillaient les cartes. Alors que tout au long du $\mathrm{XIX}^{\circ}$ siècle, l'approche juridique bénéficiait de la stabilité de la codification, le système juridique a été profondément et régulièrement modifié dès le début du $X X^{\circ}$ siècle.

Gurvitch, qui essaya de penser ces mutations et notamment le développement de structures collectives et la montée en puissance des droits sociaux, a très bien vu que la grille juridique ne permettait plus de mettre en évidence la logique cachée de ces transformations. C'est donc en partant des faits sociaux et non des textes, que Gurvitch interrogea à la suite de Levy-Bruhl, Sorokin, Simmel ou encore Duguit ${ }^{9}$, ce qui constitue la structure de la société.

Ce chemin d'exil parcouru par Gurvitch ne pouvait être naturellement suivi par la doctrine administrative française qui construisait ses frontières disciplinaires. Aujourd'hui, alors qu'on assiste à une reconstruction des savoirs sur l'administration, la question de la réalité administrative ne peut plus être mise à distance par la doctrine administrative.

II est aujourd'hui banal de constater que la logique managériale s'est largement diffusée dans le système administratif et a remodelé de nombreux

\footnotetext{
8 Jacques Caillosse, Pierre Bourdieu, Juris lectoc : anti-juridisme et science du droit, in revue Droit et société, $n^{\circ} 56-57 / 2004$, p.21.

${ }^{9}$ Voir sur ce point le chapitre « Histoire de la sociologie » rédigé par Gurvitch, dans lequel l'auteur n'hésite pas à marquer ses distances avec des auteurs aussi importants que Weber et Durkheim. Traité de sociologie, T.1. op.cit. pp.28-64.
} 
dispositifs juridiques ${ }^{10}$. L'étude de la portée de ces discours et normes sur la performance de l'administration, l'expérimentation législative ou encore l'efficacité du juge administratif ${ }^{11}$, ne peut être laissée au soin d'autres disciplines telle que la gestion ou l'économie dont les grilles d'analyses conduisent à une dissolution du phénomène juridique. En revanche, le juriste ne peut se contenter d'examiner ces phénomènes à partir de son observatoire traditionnel, l'étude de la jurisprudence, car il ne saisit qu'une strate du phénomène, une strate importante mais qui ne tient pas compte de la force de cette logique dans l'administration.

Face à cette difficulté, la microsociologie de Gurvitch peut être utile à la fois pour une meilleure définition de notre objet, tout en engageant un travail critique des discours de la dogmatique juridique.

En ce qui concerne l'objet de la science du droit administratif, Gurvitch permet d'ouvrir au droit de nouveaux territoires d'analyse en opérant un double renversement. Tout d'abord, il est un des premiers auteurs avec Hauriou, à avoir montré comment les «formes de sociabilité engendrent chacune leur propre espèce de droit » ${ }^{12}$. En forgeant le concept de «fait normatif», il renverse l'approche traditionnelle du juriste depuis les théories du contrat social, selon laquelle le droit crée l'institution pour dévoiler au contraire comment la réalité sociale est capable d'engendrer du droit ${ }^{13}$.

Dans cette perspective, l'analyse du pouvoir discrétionnaire de l'administration ne peut être saisie à partir de la simple écriture des textes et du contrôle plus ou moins étendu du juge. Gurvitch en des analyses prémonitoires, avait parfaitement senti cet enfermement, même s'il ne l'exprime pas de manière directe. Le contrôle de l'erreur manifeste d'appréciation, qui est le poste d'observation classique ne se prête pas à l'étude de la réalité juridique du pouvoir discrétionnaire. Ce dernier se retrouve dans les usages des fonctionnements des services publics et notamment dans les pratiques d'écriture des dossiers administratifs. C'est ce que montre parfaitement Axel Pohn-Weidenger dans sa thèse récente, en prenant le parti d'étudier l'action des usagers des services publics, qui bousculent les guichetiers, plaident leur cas, écrivent aux maires ou au directeur d'établissement public pour obtenir un réexamen d'un dossier ${ }^{14}$. Selon ce schéma, la norme ne vient plus seulement d'en haut, elle est travaillée par les usagers qui modifient les interprétations possibles des textes, qui raturent et annotent les dossiers et qui participent, avec les agents administratifs, à la co-écriture du document.

\footnotetext{
${ }^{10}$ Jacques Chevallier, Le droit administratif, entre science administrative et droit constitutionnel, in Le droit administratif en mutation, PUF 1993, pp34-40.

${ }^{11} \mathrm{Cf}$. notamment l'ouvrage collectif, L'efficacité de la justice administrative, à la recherche d'une nouvelle légitimité, (dir. M. Lavaine et R. Matta-Duvignau), ed. Mare et Martin 2016.

${ }^{12}$ Cf. G.Gurvitch, Eléments de sociologie juridique, (1940), éd. Dalloz 2012, p.144.

${ }^{13}$ Cf. G.Gurvitch, Eléments de sociologie juridique, op.cit.p.144. Pour une étude récente par la doctrine administrative voir par exemple, l'ouvrage dirigé par J-M. Pontier et E.Roux, Les faits en droit administratif, éd. PUAM 2010.

${ }^{14}$ Axel Pohn-Weidenger, Ecrire dans les plis du droit social, une sociologie du dossier, Thèse dactylographiée, Paris 8, 2014.
} 
Ce changement d'échelle conduit à une autre cartographie de la normativité administrative que le Conseil d'Etat, lui-même, commence à prendre en compte en s'intéressant sérieusement à des productions textuelles de l'administration qui peuvent prendre la forme de communiqués de presse, ou de prises de position d'autorités publiques. Dans deux décisions récentes le juge administratif a accepté des recours en annulation contre ce qu'il appelle du "droit souple", qui traditionnellement échappait à son contrôle ${ }^{15}$. En réalité, il ne fait qu'accentuer sa surveillance en examinant désormais les documents dits non-normatifs, mais qui dirigent réellement les comportements et les conduites des agents. Certains considèrent qu'avec cette évolution le droit perd en précision mais le juge nous invite seulement à nous intéresser plus aux significations des énoncés juridiques interprétés par les acteurs, qu'aux formes juridiques, de plus en plus vides de sens.

Ce changement d'objet conduit par contre coup à un travail de déconstruction. A la suite d'auteurs aussi différents que Duguit ou encore Bergson, Gurvitch nous incite à engager un travail de "démolition de tout concept momifié, de tout cadre opératoire rendu immobile, de toute expérience arrêtée ${ }^{16}$. Cette démarche aujourd'hui résonne tout particulièrement. D'une part, de manière générale, le concept est devenu, avec la philosophie du langage, un des points clefs de toute pensée. Et d'autre part, en droit et plus particulièrement en droit administratif, les concepts de service public, de puissance publique, de consentement, d'aléa, d'actes juridictionnels jouent comme des masses immobiles, posées dans le discours juridique. Or, pour saisir le fait normatif administratif, il faut s'émanciper des concepts formels pour atteindre la réalité juridique administrative.

Ainsi par exemple, l'acte administratif unilatéral qui sert généralement de pilier aux juristes administratifs dans ses constructions doctrinales, abrite des réalités juridiques très différentes. En effet, le concept d'acte administratif unilatéral est généralement défini comme l'acte qui fait naître des droits ou crée des obligations, indépendamment de la volonté de son destinataire. Ce découpage de la réalité conçoit l'acte unilatéral comme "l'acte d'un seul côté ${ }^{17}$ » mais la réalité juridique est bien plus complexe car le juriste administratif sait bien que de nombreux actes unilatéraux sont négociés, discutés, élaborés collectivement. L'approche réaliste, qui s'appuie sur la théorie des actes de langage, montre par ailleurs que l'unilatéralité pure n'existe pas car le texte est "en tant que tel pragmatiquement muet ${ }^{18}$ car seule la lecture du texte produit ou ne produit pas la norme juridique. Le fait administratif unilatéral est bien différent de la représentation idéale véhiculée par les définitions officielles.

Le droit administratif pourrait fournir de nombreux exemples de ce processus de réduction. La doctrine a engagé un travail de longue date d'élaboration de

\footnotetext{
${ }^{15}$ Cf. CE 21 mars 2016, Société Faivesta International GMBH et autres; CE 21 mars 2016, société NC numéricable.

${ }^{16}$ G.Gurvitch, La vocation actuelle de la sociologie, PUF 2é p. 488

${ }^{17}$ C.Eisenmann, Cours de droit administratif, réed LGDJ, col. Anthologie du droit, 2014.

${ }^{18}$ Olivier Cayla, Lire l'article 55 de la Constitution : comment comprendre un texte établissant une hiérarchie des normes comme étant lui-même le texte d'une norme ? Les cahiers du Conseil constitutionnel, $n^{\circ} 7$,
} 
concept pour se dégager de la réalité empirique. Cette réalité empirique administrative apparait comme un maquis touffus, mis en ordre régulièrement ${ }^{19}$ par des juristes soucieux de faire apparaitre des unités intelligibles qui transcendent les particularités et les différences. Avec Gurvitch, la richesse de la matière administrative est à nouveau le point de départ de l'analyse. Une telle approche ouvre des perspectives qui conduisent à l'abandon d'un certain nombre de postulats.

Postulat de la localisation du droit administratif : traditionnellement, le droit administratif est représenté comme un droit produit par l'Etat qui trouve sa source dans le pouvoir réglementaire du Premier ministre et du président de la république et qui se diffuse au sein de l'appareil étatique. Une grande partie des concepts de la théorie juridique de l'Etat participe à la construction de cette représentation qui donne à voir un espace juridique unifié. Mais l'analyse juridique montre bien la complexité du système lorsqu'elle ne veut pas passer à côté de la multiplicité des rouages et des institutions producteurs de droit, qui se situent tous à des niveaux différents ${ }^{20}$. Dans cette perspective, la question de la réforme territoriale ne peut se réduire au schéma centre-périphérie ou encore unité/décentralisation et doit tenir compte de la multiplicité des institutions, nationales/locales mais aussi publiques/privées qu'on rencontre sur le terrain.

Postulat de la neutralité du droit administratif : le droit administratif serait neutre axiologiquement. II serait dégagé de toute idéologie, si ce n'est peut-être la notion d'intérêt général, qui embarrasse la doctrine depuis presque deux siècles. II faut penser le droit administratif comme un ensemble de technique qui se situerait en aval du politique. Cette représentation d'un droit administratif comme droit d'exécution ou encore droit de concrétisation, est bien sûr en décalage avec les analyses de politiques publiques et les études de terrain qui permettent une autre identification des circuits de décision. Cette dernière est soumise à des conditionnements multiples et obéit rarement à ces schémas séquentiels.

Postulat de l'objectivité du droit administratif comme donné : au bout du compte, l'apport essentiel d'une lecture de Gurvitch est d'avoir montré que les problèmes ne se posent pas d'eux-mêmes et que le droit n'est pas un simple donné. II explique à propos de la morale que la première tâche de la sociologie est précisément "cette construction des faits ${ }^{21}$ à partir de la réalité sociale brute. C'est le travail d'objectivivisation, inscrit dans un cadre social, qui conduit à l'objet. La doctrine administrative est elle-même une production sociale, tributaire de codes, de rituels qui font la culture juridique.

Cette rupture épistémologique, aussi difficile soit-elle, pourrait permettre d'être au plus près de la matière juridique. Reste la difficulté d'aller contre une manière dominante de penser le juridique qui se présente comme naturel. Gurvitch avait mesuré l'obstacle parfaitement mais auteur iconoclaste, il n'avait pas peur du conflit. Conflit qui est au cœur de sa pensée.

\footnotetext{
${ }^{19}$ Sur ce point, on peut évoquer le travail annuel de l'Association Française pour la recherche en Droit Administratif qui a entrepris un travail de réélaboration des concepts fondateurs de la discipline. Voir par exemple, Le service public, éd. Dalloz, col. Thèmes et commentaires, 2014.

${ }^{20}$ Sur ce point, voir Jean-Marie Pontier, La problématique du territoire et du droit, in Droit et complexité, (dir. M.Doat, J. Le Goff et P.Pedrot, éd. PUR 2007, pp.39-62.

${ }^{21}$ G. Gurvitch, Morale théorique et science des mœurs, PUF 1948, p.202.
} 


\section{II - Du conflit administratif : le pluralisme juridique}

En manière administrative, et plus généralement dans les rapports entre l'Etat et les administrés, l'idée même de conflit n'a pas de place dans l'analyse juridique. Des théories de l'Etat au contentieux administratif, le langage administratif a fait disparaître le terme du lexique juridique. Dans le discours libéral, les idéaux de transparence, de participation, d'intégration, de délibération, permettent d'éradiquer l'idée d'opposition entre personnes ou entre institutions. Ils permettent de refouler les colères, la frustration, la rancune ou encore l'agressivité des relations administratives.

Le tour de passe-passe est dangereux car, comme l'a montré Gurvitch, en niant les conflits nés de la multiplicité, on met en danger le fonctionnement de la société. Dans son parcours, on sait que Gurvitch a commencé très tôt à lire les auteurs critiques comme Marx, Karl Kautsky, Proudhon ou encore Lénine ${ }^{22}$. Les transformations, révolutions, guerres, crises sociales et économiques sont en toile de fond de son cheminement. Analysant le pluralisme juridique mais aussi le cadre social de la connaissance, il a été un des rares auteurs de formation juridique à mettre au jour les dérives du capitalisme. II démonte aussi bien les illusions de la société unifiée que celle de la " connaissance universelle ».

En faisant du pluralisme juridique le point de départ de son analyse, il conçoit le système juridique comme un espace d'affrontement. Ainsi, dans «La déclaration des droits sociaux ", il indique clairement que "toute déclaration des droits comme tout devoir être et toute norme, est d'abord dirigé contre quelque chose $»^{23}$. Dans un espace pluriel, la norme se déploie par opposition et par contradiction. Elle n'est pas la concrétisation d'une norme supérieure pour reprendre le schéma kelsennien qui conçoit l'ordre juridique comme un ensemble cohérent, monolithique. Cette norme se construit par opposition à une autre norme : la norme locale contre la norme nationale, la norme publique contre la norme privée, la norme générale contre la norme particulière. Ces obstacles sont internes au système juridique mais aussi externe.

Dans des analyses qui résonnent particulièrement aujourd'hui, Gurvitch, montre notamment comment le premier obstacle que rencontrent les droits sociaux se trouve dans le développement d'une nouvelle économie qui forme " un nouveau gouvernement privé » qui fait "surgir de vastes organisations de domination autoritaire, échappant à tout contrôle autoritaire " et "empêche par le mur de l'argent toute réforme indispensable $\aleph^{24}$. C'est contre le développement de la technologie que doit se dresser un droit social «fondé sur la confiance et la participation $»^{25}$, émergeant de l'institution.

\footnotetext{
${ }^{22}$ Cf. M.Antonov et E.Berthold, Sources russes de la pensée de Georges Gurvitch : écrits de jeunesse dans les annales contemporaines (1924-1931), Cahiers internationaux de sociologie, 2006, p.197 \& s.

${ }^{23}$ G.Gurvitch, La déclaration des droits sociaux, op.cit. p.48.

${ }^{24}$ G.Gurvitch, La déclaration des droits sociaux, op.cit. p.49.

${ }^{25}$ G.Gurvitch, La déclaration des droits sociaux, op.cit. p.76.
} 
Aujourd'hui, la lecture de Gurvitch est tout à fait édifiante. La doctrine administrative est confrontée à un paysage juridique "bouleversé, où les règles de droit semblent surgir de partout, à tout moment et en tout sens ${ }^{26}$. L'Etat est pris dans un système complexe, construit d'institutions locales ou européennes qui agissent simultanément. Les thèses du pluralisme juridique, qui avaient le statut d'oubliées et étaient laissées aux analyses de la seule sociologie, sont convoquées pour décrire ces transformations ${ }^{27}$. Mais si la doctrine porte un regard différent sur le système juridique moderne, elle éprouve des difficultés pour expliquer les rapports interinstitutionnels et essaye toujours de donner à voir un ordre juridique harmonieux, construit plus sur la diversité que sur l'unité. II s'agit de maintenir une image apaisante, grâce à aux images du "dialogue des juges " ${ }^{28}$ et de la bonne administration de la justice. La métaphore botanique du rhizome ${ }^{29}$ est mise en avant pour l'opposer à la rigidité de la pyramide, mais le champ lexical reste rassurant.

Tout au long de la deuxième partie de 20ème siècle, on a assisté à une simplification lexicale et syntaxique de la langue administrative destinée à rendre impossible l'expression des idées potentiellement subversives de pouvoir et de domination et à éviter toute formulation de critique de l'État, l'objectif ultime étant d'aller jusqu'à empêcher l'« idée » même de cette critique. Daniele Lochak a parfaitement montré comment pendant la seconde guerre mondiale, la banalisation du droit antisémite a été opérée par la mise en place d'un voile de concepts juridiques abstraits ${ }^{30}$. Elle a remarquablement poursuivi ce travail pour décrire l'effet de "déréalisation et d'euphémisation » du discours juridictionnel, notamment en matière de droit des étrangers ${ }^{31}$. Le requérant est en effet en partie dessaisi du conflit qui l'oppose à l'administration. II n'agit pas contre une personne mais selon la formule habituelle, il fait " un procès à un acte ». Le conflit devient contentieux, mot qui se transforme en litige au cours du procès. L'échange avec l'administration se fait dans le cadre d'une procédure écrite et d'un échange de mémoire....

Ce resserrement du langage se constate dans d'autres domaines, et peut être parfois le résultat de l'action du législateur. C'est le cas notamment en matière de décentralisation, lorsque la loi du 2 mars 1982 a supprimé le mot de tutelle pour le remplacer par celui de contrôle administratif. Si juridiquement le contrôle des préfets reste encore aujourd'hui un contrôle que l'on peut qualifier de tutelle, le mot n'existe plus dans le champ du discours sur la décentralisation.

\footnotetext{
${ }^{26}$ M.Delmas-Marty, Pour un droit commun, éd. du Seuil, 1994, p.52.

27 Voir par exemple, l'ouvrage collectif dirigé par Laureline Fontaine, dans lequel le concept de pluralisme juridique est mobilisé par des juristes pour décrire différentes transformations. Droit et pluralisme, éd. Bruylant 2007.

${ }^{28}$ Sur cette expression, on trouve de très nombreuses communications notamment des institutions juridictionnelles. Inventée par Bruno Genevois, ancien président de la section contentieuse du Conseil d'Etat, l'expression est devenue un élément du langage afin de désigner la collaboration et l'écoute des juges issus de différents niveaux de juridiction.

${ }^{29}$ Sur la banalisation de cette métaphore, voir F.Ost et M. Van de Kerchove, De la pyramide au réseau, Publication des Facultés universitaires de Saint-Louis, 2002.

${ }^{30}$ Daniel Lochak, La doctrine sous Vichy ou les mésaventures du positivisme, in Les usages sociaux du droit, (dir. D. Lochak), PUF 1989, pp.252-285.

${ }^{31}$ Daniel Lochak, Dissimuler la violence, canaliser la contestation, in L'office du juge, éd. du Sénat, 2006, p.248.
} 
Enfin, il serait possible de voir comment le discours administratif s'est construit autour d'un certain nombre de dichotomies (service public/puissance publique, intérêt général/intérêt des particuliers, responsabilité pour faute/sans faute, loi/règlement, acte unilatéral/contrat...) qui suppriment toutes les nuances de la réalité administrative et éliminent du même coup toute réflexion sur la complexité d'un problème. Ce discours manichéen permet d'éliminer toute identification du conflit.

Cette interprétation apaisante du pluralisme juridique apparaît donc trop simple et la lecture de Gurvitch permettrait d'enrichir le lexique pour décrire le « champ de bataille ${ }^{32}$, les trahisons, les obstacles et les rapports de force produits par le système juridique.

C'est aussi un des apports de Gurvitch d'avoir utilisé une rhétorique énonciatif et argumentatif dont la visée pragmatique est la disqualification de la cible et la mise à mort de l'adversaire. Auteur polémique, on peut lui appliquer les mots de Bachelard lorsqu'il retrace l'histoire de la sociologie: " on connait contre une connaissance antérieure, en détruisant des connaissances mal faites ${ }^{33}$. C'est en contredisant les pères de la discipline comme Durkheim ou Weber que sa pensée s'est construite ${ }^{34}$. Cet édifice verbal peut paraître daté aujourd'hui et il est certain qu'il est en décalage aujourd'hui avec les discours plus mesurés des échanges scientifiques. Avec Gurvitch, on était dans le combat, la mêlée, un art raffiné de la dialectique, construit sur des agencements conflictuels. II y a dans ces textes une part de ruse, une grande maîtrise, une vraie spontanéité, un engagement passionnel. Nul doute que sa lecture reste passionnante.

\footnotetext{
32 G.Gurvitch, op.cit. p.53

${ }^{33}$ G.Bachelard, Le nouvel esprit scientifique, (1934) $13^{\circ}$ éd Vrin, p.14.

${ }^{34}$ Voir sur ce point son histoire de la sociologie dans le traité qu'il a dirigé : Traité de sociologie, PUF 1958, pp.28-64.
} 